\title{
Machine Learning Guided 3D Image Recognition for Carbonate Pore and Mineral Volumes Determination
}

This paper was downloaded from TechRxiv (https://www.techrxiv.org).

\section{LICENSE}

CC BY 4.0

SUBMISSION DATE / POSTED DATE

$09-11-2021 / 11-11-2021$

\section{CITATION}

Alfarisi, Omar; Raza, Aikifa; Zhang, Hongtao; Sassi, Mohamed; Zhang, TieJun (2021): Machine Learning Guided 3D Image Recognition for Carbonate Pore and Mineral Volumes Determination. TechRxiv. Preprint. https://doi.org/10.36227/techrxiv.16961551.v1

$\mathrm{DOI}$

10.36227/techrxiv.16961551.v1 


\begin{tabular}{|l|l|l|}
\hline \multicolumn{2}{|l|}{ Title } & $\begin{array}{l}\text { Machine Learning Guided 3D Image Recognition for Carbonate Pore and Mineral } \\
\text { Volumes Determination }\end{array}$ \\
\hline \multicolumn{2}{|c|}{ Surname } & \multicolumn{1}{c|}{ Company } \\
\hline Given Name & \multicolumn{1}{|c|}{ Al-Farisi } & $\begin{array}{l}\text { Khalifa University of Science and Technology \& } \\
\text { Abu Dhabi National Oil Company Offshore }\end{array}$ \\
\hline Omar & Raza & Khalifa University of Science and Technology \\
\hline Aikifa & Zhang & Khalifa University of Science and Technology \\
\hline Hongtao & Ozzane & Abu Dhabi National Oil Company \\
\hline Djamel & Sassi & Khalifa University of Science and Technology \\
\hline Mohamed & Zhang & Khalifa University of Science and Technology \\
\hline TieJun & & \\
\hline & & \\
\hline
\end{tabular}

\section{Abstract}

Automated image processing algorithms can improve the quality, efficiency, and consistency of classifying the morphology of heterogeneous carbonate rock and can deal with a massive amount of data and images seamlessly. Geoscientists and petroleum engineers face difficulties in setting the direction of the optimum method for determining petrophysical properties from core plug images of optical thinsections, Micro-Computed Tomography $(\mu \mathrm{CT})$, or Magnetic Resonance Imaging (MRI). Most of the successful work is from the homogeneous and clastic rocks focusing on 2D images with less focus on 3D and requiring numerical simulation. Currently, image analysis methods converge to three approaches: image processing, artificial intelligence, and combined image processing with artificial intelligence. In this work, we propose two methods to determine the porosity from 3D $\mu \mathrm{CT}$ and MRI images: an image processing method with Image Resolution Optimized Gaussian Algorithm (IROGA); advanced image recognition method enabled by Machine Learning Difference of Gaussian Random Forest (MLDGRF).

Meanwhile, we have built reference 3D micro models and collected images for calibration of the IROGA and MLDGRF methods. To evaluate the predictive capability of these calibrated approaches, we ran them on 3D $\mu \mathrm{CT}$ and MRI images of natural heterogeneous carbonate rock. We also measured the porosity and lithology of the carbonate rock using three and two industry-standard ways, respectively, as reference values. Notably, IROGA and MLDGRF have produced porosity results with an accuracy of $96.2 \%$ and $97.1 \%$ on the training set and $91.7 \%$ and $94.4 \%$ on blind test validation, respectively, in comparison with the three experimental measurements. We measured limestone and pyrite reference values using two methods, X-ray powder diffraction, and grain density measurements. MLDGRF has produced lithology (limestone and pyrite) volume fractions with an accuracy of $97.7 \%$ in comparison to reference measurements.

\section{Introduction}

It is challenging to have static and dynamic reservoir properties determination and integration from micro and nano CT images with machine learning aided image recognition (Dietterich 2000, Decencière et al. 2013, Arganda-Carreras et al. 2017, Sommer et al. 2011). The current literature review has revealed the pressing challenges regarding the need to perform 3D image processing instead of 2D (Sánchez et al. 2008, Li and Chutatape 2004, Bovik 2009). Dual-energy $\mu$ CT scanning data is used to characterize the rock properties (Saxena et al. 2017, Dernaika et al. 2018, Rafael et al. 2007), and claimed reasonable matching between measured core plug helium porosity, calculated using Digital Rock Physics (DRP) methods. The only drawback is lack of blind test; instead, the work appeared as a learning and exploring $\mu \mathrm{CT}$ abilities to deliver quality images for rock characterization. (Dernaika et al. 2018) Work suggested that a 2D image cannot predict the petrophysical properties in carbonate due to the limited representation of heterogeneous rock in 2D. Lately, major oil operator (Chhatre et al. 2017) stated that no commercial vendor is yet able to measure the porosity and permeability.

Besides, carbonate is harder. In work performed (Chhatre et al. 2017) to check four DRP vendors' capabilities, the findings suggest no vendor managed to get Porosity, Permeability, and $P_{c}$ (Capillary 
Pressure) curves correctly. The work also recommended that future DRP analysis should also report the uncertainties. Artificial Intelligence (AI), mainly machine learning, was used to characterize rock properties (Alqahtani et al. 2018) by analyzing $\mu \mathrm{CT}$ images, where segmentation is a method for porosity calculation. A Convolutional Neural Network $(\mathrm{CNN})$ was used to get the pore size distribution. The use of 2D images (Alqahtani et al. 2018), reminding us of the thin-sections analysis, and highlighting the effect of vugs that acts as large channels or high connectivity between matrix porosity, where the reported analysis is made using open-source ImageJ software (Collins 2007, Abràmoff et al. 2004) and Tensor Flow software (Abadi et al. 2016). While 3D Image analysis (Dong and Blunt 2009) for $\mu$ CT images followed an approach called Maximal Ball algorithm, as topological extraction of the pore network is the central part. The primary importance (Dong and Blunt 2009) is the estimation of the connectivity between each pore, knowing that quantifying connectivity is essential to calculate rock dynamic properties in putting a way forward an estimator for capillary pressure and relative permeability. NMR is a preferable porosity logging measurement in the oil industry (Xu et al. 2015), as it is lithology independent. For a fully saturated core plug (like oil or water), the NMR signal represents rock total porosity. While if water (or oil) saturated core contains gas, then the gas is invisible to NMR. So, in the case of $\mathrm{CO}_{2}$ flooding, the NMR will act as a liquid saturation tool. In (Liu et al. 2016), work focused on NMR for saturation measurement, the relation between relaxation time and pore size (Ausbrooks et al. 1999) was an evident link. Other research (Marschall et al. 1995) earlier used the same methodology.

In this work, therefore, we focused on Magnetic Resonance Imaging (MRI) to determine porosity and on $\mu \mathrm{CT}$ to determine both porosity and lithology. As discussed earlier, carbonate is complex to characterize using image analysis (Saxena et al. 2017) for determining porosity and permeability, concluding that image processing suits clastics only due to low heterogeneity. We have realized that tackling our technical challenge of determining porosity and lithology in heterogeneous rock requires an interdisciplinary approach, an amalgamated knowledge of fluid dynamics, reservoir characterization (rock typing, and petrophysical analysis), image processing, machine learning, and software coding is vital to deliver the solution. Our research deployed new analysis and validation approaches, including building reference 3DMM (3D Micro Model) with various micropore sizes and use them as image processing calibration jig. Besides, we developed a new image resolution enhancement algorithm for high-quality segmentation. In our paper, we determined porosity using mainly two methods. The first is a standalone image processing, where image feature extraction was successful. The second is the machine learning assisted image recognition. These methods depend on the training data from experimental results. We define Machine Learning as the ability of a machine to perform the learned tasks, as good as the training set, on new data, faster and more accurate than a human expert, while the machine is still not smarter than humans when the context is unfamiliar for the machine (Phillips and O'toole 2014). In other words, human indexing of information appears to be multi-contextual adaptability, while machine learning is a single context or contextually limited (Whitney 2017). The machine performs specific tasks more effortless than a human can perform, and humans perform specific tasks more comfortable than a machine, including image recognition (Goodfellow 2016) which is easier for humans than a machine. The machine can perform repetitive, rule-based mathematical tasks, but lacks creativity and intuition (Whitney 2017, Marr 2016, August 12). In more engineering sense, the machine learning definition stepwise is; human expert input training data to machine and selects learning algorithms; the machine performs the learning to build and test a model, then use the model to perform prediction on new data set, also known as supervised machine learning (Kotsiantis et al. 2007). Below we give a background on the concepts investigated, which are; machine learning, image processing, and combined machine learning and image processing.

\section{Preliminaries}

Coupling the latest science, engineering, and technology advancement became a necessary tool to solve long-standing challenges of rock properties characterization from $3 \mathrm{D}$ microimages; $\mu \mathrm{CT}$ and MRI. 
In this section, we provide a brief description of the coupling between machine learning and image processing, starting by introducing each concept individually and then the combination of both, as per the following:

\section{1- Machine Learning}

The utilization of machine learning capabilities is vital for quality and efficiency, as the machine can reproduce results by mimicking what domain expert has taught. We have followed four steps in deploying machine learning:

(1) Step 1: Human expert (Geoscientist), a Petrophysicist in our case, provided data set to the machine. Our petrophysicist identified two chemical (mineral) properties (Limestone and Pyrite), two physical properties (pore and solid), and various image intensities (Chen et al. 1998, Collewet et al. 2004, Shattuck et al. 2001, Wang, Aghaei, et al. 2017) corresponding to limestone, pyrite, and pore. The data set contains:

i. "Independent parameters data columns"; image identified properties with black color, dark gray intensity, light gray intensity, and white.

ii. "dependent data column,"; containing the desired classes of limestone, pyrite, and pore, while Solid here represents an aggregation of both lithology types; limestone and pyrite.

(2) Step 2: Human Expert chooses an open-source Machine Learning Algorithm (Pedregosa et al. 2011); in our case, we used the "Random Forest" algorithm (Breiman 2001), owing to its superior capability in performing classification.

(3) Step 3: The computer (machine) uses the Random Forest algorithm to learn from the data set and build the prediction model with all its governing equations ( $\mathrm{Yu}$ et al. 2016), where the machine develops by itself the required formulas that enable solving the assigned problem.

(4) Step 4: The computer uses the input data set, 3D $\mu \mathrm{CT}$ and MRI image stack, and deploys the learned prediction model with its governing equations to predict output classes.

\section{2- Image Processing}

We define image processing as a task human expert performs on the image (2D or 3D) that produces a new set of information or a new image version to provide more insight. In more engineering sense, the image processing definition employed stepwise (Russ 2016) as per the following:

(1) Step 1: Input an image (2D, 3D) to a machine. In our research, we focused on 2D and 3D images only. Where 2D represents surface in gray or color. While 3D represents the volumetric view (Zhang et al. 2006, Deriche 1987, Dunlavy et al. 2008, Calliess et al. 2012).

(2) Step 2: Domain expert runs suitable filtering, segmentation, and analysis algorithms (Malcolm et al. 2007, Lindquist et al. 1996, Ehrlich et al. 1984, Lindquist and Venkatarangan 1999, Mowers and Budd 1996) to achieve the image-processing objectives.

(3) Step 3: The human expert learns new insight and generates new data (numerical or image) from the above step.

\section{3- Combining Machine Learning and Image Processing}

The combination scenario called Machine Learning Image Recognition (MLIR), which is defined as "the ability of a machine to perform tasks" (Kann et al. 2019) as good as training set, image interpretation, delivered by a human expert, on new images faster and more accurate than human expert but not smarter than a human expert. Another definition also describes machine learning and image processing (Gonzalez et al. 2009). Image recognition machine learning (He, Zhang, et al. 2016, Simonyan and Zisserman 2014) requires a longer processing time in comparison with the processing time needed in performing image processing alone. 


\section{Problem Articulation}

Proper image classification (segmentation) of variant rock sections enables the quantification of its properties. However, segmentation accuracy depends heavily on how representative the image is to a real object. The pore sizes of our rock are either at or below the resolution of the image voxel size. In the following sections, we describe two challenges that we faced with $3 \mathrm{D} \mu \mathrm{CT}$ and MRI images:

\section{1- First Challenge: Resolution Effect on 3D $\mu$ CT Images}

Image resolution (Yang et al. 2010) is one of the main challenges in the 3D morphological (Huang et al. 2008) recognition process. Naturally, better resolution leads to better segmentation (Drexler et al. 2001, Schindelin et al. 2012). However, not every image comes with the desired resolution for optimal image analysis. In 3D rock images, identifying $10^{6}$ of micro-pores and quantifying their morphology (Gerke et al. 2015, Ehrlich et al. 1984, Singh et al. 2017) are pressing challenges. The resolution does blur the feature of interest. Numerically, in blurring condition, the pixel color value does not represent the actual color value for that position in space. We acquired 3D $\mu \mathrm{CT}$ images for dry (pore contains air only) carbonate rock and displayed one of its 2D slices in Fig. 1, where we see how micro-pores in carbonate rock appear in more than one gray value, although it should appear as black. The cause of this challenge is low resolution, where the pore size is smaller than the pixel resolution that used to acquire the image. The pore must be black color, where the pixel should hold a value of " 0 ". To accurately measure porosity and lithology (rock minerals), we must correct images to be " 0 " value for pore; otherwise, it is the rock-solid. The challenge is how to do correction without mistakenly converting true-non-pore to a pore. In Fig. 1, the top image shows a $38 \mathrm{~mm}$ diameter sample acquired with $40 \mu \mathrm{m}$ resolution (each pixel is $40 \mathrm{x} 40 \mu \mathrm{m}$ ). The image inside the red circle is our zone of interest for identifying pore (Black) and solid (Light color or non-black). To solve the resolution effect, we used a convolutional Gaussian kernel for improving the resolution of 3D images.

\section{2- Second Challenge: Resolution Effect on 3D MRI images}

To identify the fluid distribution in the pore spaces, MRI can be one of the preferable options (Wang, Lun, et al. 2017, Zhou et al. 2001). We used the Suzhou NiumagTM NMR system (0.5 T). Besides, we proved, that MRI can also act as a porosity measuring tool independent of lithology type for saturated rock with single-phase fluid. The same carbonate rock sample was used to acquire 3D MRI images, as shown in Fig. 2. The saturated pores appear a lighter color in MRI, while solid appears as dark (Black). We flooded the pores of a rock sample with crude oil. Fig. 2 shows three MRI cross-section images acquired with three different sampling rates and different slice thickness along the $\mathrm{z}$-axis. Image voxel (x, $\mathrm{y}, \mathrm{z})$ resolution is $(400 \times 400 \times 3500 \mu \mathrm{m})$ for Fig. 2a. While it is $(400 \times 400 \times 2500 \mu \mathrm{m})$ for Fig. $2 \mathrm{~b}$ image. And it is $(400 \times 400 \times 1500 \mu \mathrm{m})$ for Fig. 2c. For porosity determination, and for fluid monitoring, it is important bringing Fig. 2c image (thinner 2D MRI slice, or higher resolution in the z-axis) to be clear as Fig. 2a image (thicker 2D slice or lower resolution in the z-axis). At the same time, we must improve the resolution of Fig. 2a as good as Fig. 2b. We can notice that Fig. 2a has a higher dynamic range of intensity despite its lower resolution (due to thicker sampling size along the z-axis). 


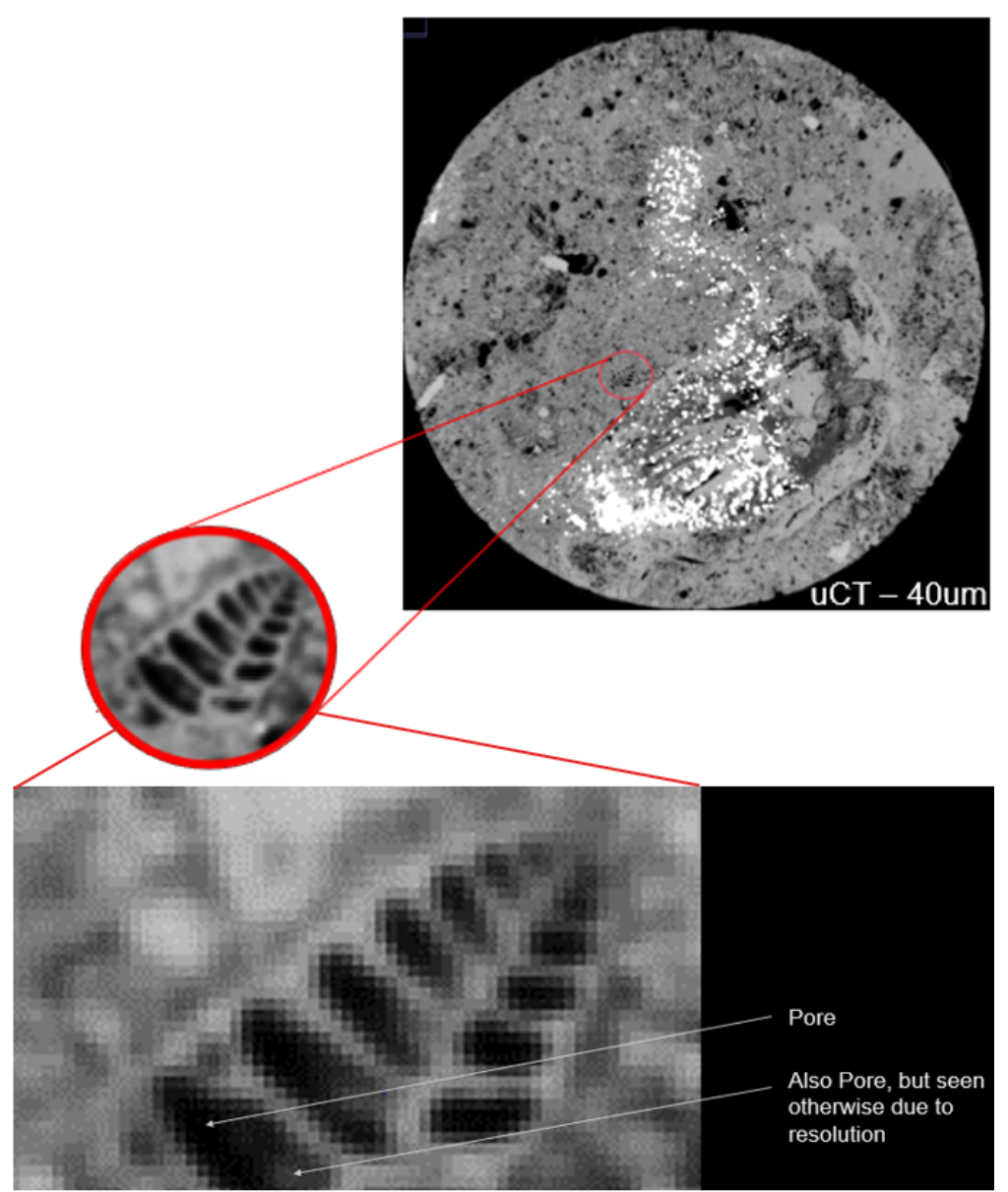

Fig. 1-The resolution effect is evident in three different zooming scales.

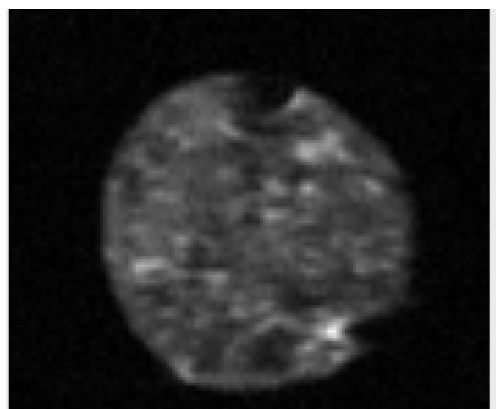

(a)

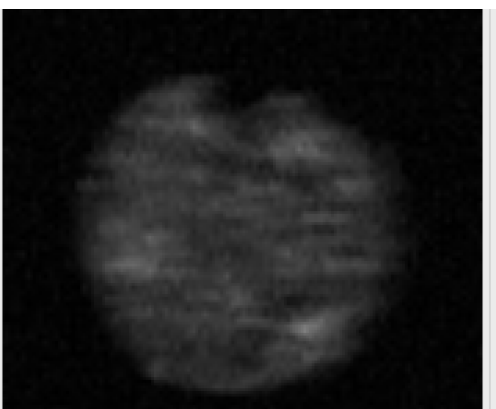

(b)

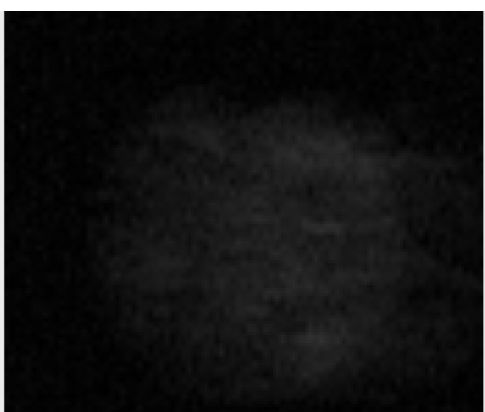

(c)

Fig. 2-MRI (Magnetic Resonance Imaging) images for a carbonate core plug with three different sampling resolutions.

\section{Proposed Methods}

We focused on two methods for solving $\mu \mathrm{CT}$ and MRI images resolution to enable further analysis of these images to extract petrophysical properties. To achieve resolution enhancement and faster performance at the same time, we chose image processing as a starting option. Then we compared the image processing results with what the machine learning approach can deliver. So, we conducted an experimental screening of two different methods. The sequence of our work in developing the best methodology for determining porosity and lithology is as described below:

\section{1- First Method: Image Resolution Optimized Gaussian Algorithm (IROGA), an Image Processing Algorithm}

A universal image enhancer provided an easy way for processing future MRI images without the need for human expert labeling; that is what we thought and then innovated and described in this paper. To 
address the resolution effects, we launched an experimental task that involves building a 3D Micro Model (3DMM). Using 3DMM MRI images as a calibration reference enabled us producing universal image resolution enhancement, which is usable for similar acquisition devices, temperature, and acquisition parameters. Therefore, for a different device, environment, and acquisition parameters, the same methodology we explain below is viable after conducting a new calibration run. We fabricated several micro models to quantify the blurriness of the image and calibrate it. In this paper, we described one of the 3DMM briefly, which is the bundle of capillary tubes 3DMM. To increase the resolution by reducing the blurriness effect, we needed to build an image correction model, and at the same time, we had to ensure model performance efficiency. Therefore, a convolutional Gaussian image processing filter (Li et al. 2018, Buades et al. 2005) was the first choice to achieve better resolution. After several trial and error rounds, we managed to develop a novel optimized convoluted Gaussian image processing algorithm, IROGA (Image Resolution Optimized Gaussian Algorithm). So, based on 3DMM MRI images, we constructed IROGA, as shown in Fig. 3. While segmenting (or interchangeably, we use "classifying") more than two classes, machine learning algorithms like the random forest (Sommer et al. 2011) or others would be more suitable. We also used the words segments, labels, classes, clusters interchangeably as they mean the same in this paper. Let us keep in mind that IROGA as a standalone algorithm is useful when we target one or more of the following goals:

i. Enhance the resolution of an image.

ii. Differentiate two labels (binary).

iii. Perform points (i) and (ii) above together.

iv. Determine porosity from a low-resolution image.

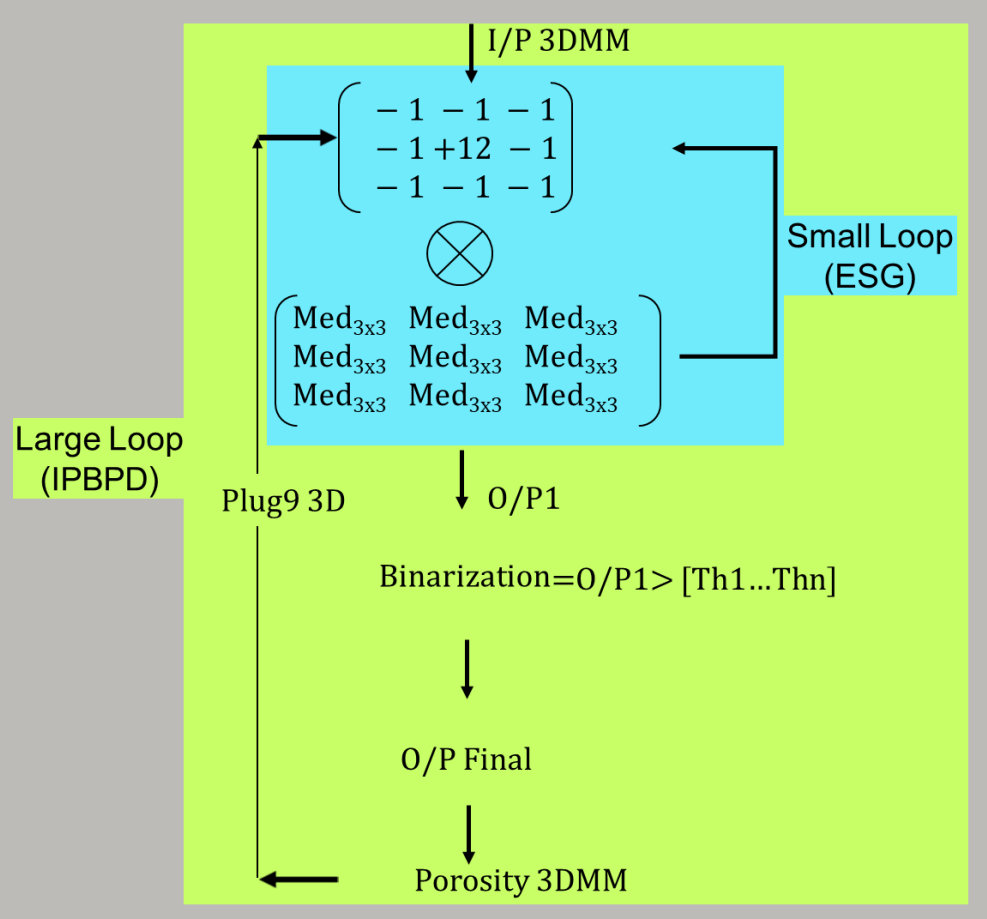

Fig. 3-IROGA (Image Resolution Optimized Gaussian Algorithm) Block Diagram. Small Loop is also called Enhanced Convoluted Gaussian. Large Loop is also called Image Processing Based Porosity Determination.

\section{2- Second Method: Machine Learning Image Recognition (MLIR)}

In the machine learning method, we performed $3 \mathrm{D} \mu \mathrm{CT}$ image recognition using the Machine Learning Difference of Gaussian Random Forest (MLDGRF) algorithm. The primary machine learning performed tasks were as described earlier in the introduction section. The specific steps for porosity and lithology determination are; human expert provided pore, solid (limestone and pyrite) labels, and selected the 
Random Forest algorithm to let the machine learn and then predict the segments. To do so, we used mainly three software; Microsoft Office 365 ${ }^{\mathrm{TM}}$ Excel (Wilson 2014), Python (Müller and Guido 2016), and ImageJ (Ferreira and Rasband 2012) for machine learning, image processing, mathematical modeling, and graph generation. Fig. 4 shows schematics of our proposed method of Machine Learning Image Recognition (MLIR), by describing gradual composing of MLIR in three steps; identify optimal Machine Learning algorithms, deploy image recognition for targeted image feature; where image recognition is a branch of image processing (Gonzalez et al. 2009). Then we combine both machine learning and image processing. Fig.5 shows the MLIR algorithm description. Besides, to see the difference between Gaussian and Difference of Gaussian Function, we plotted both functions in Fig. 6.

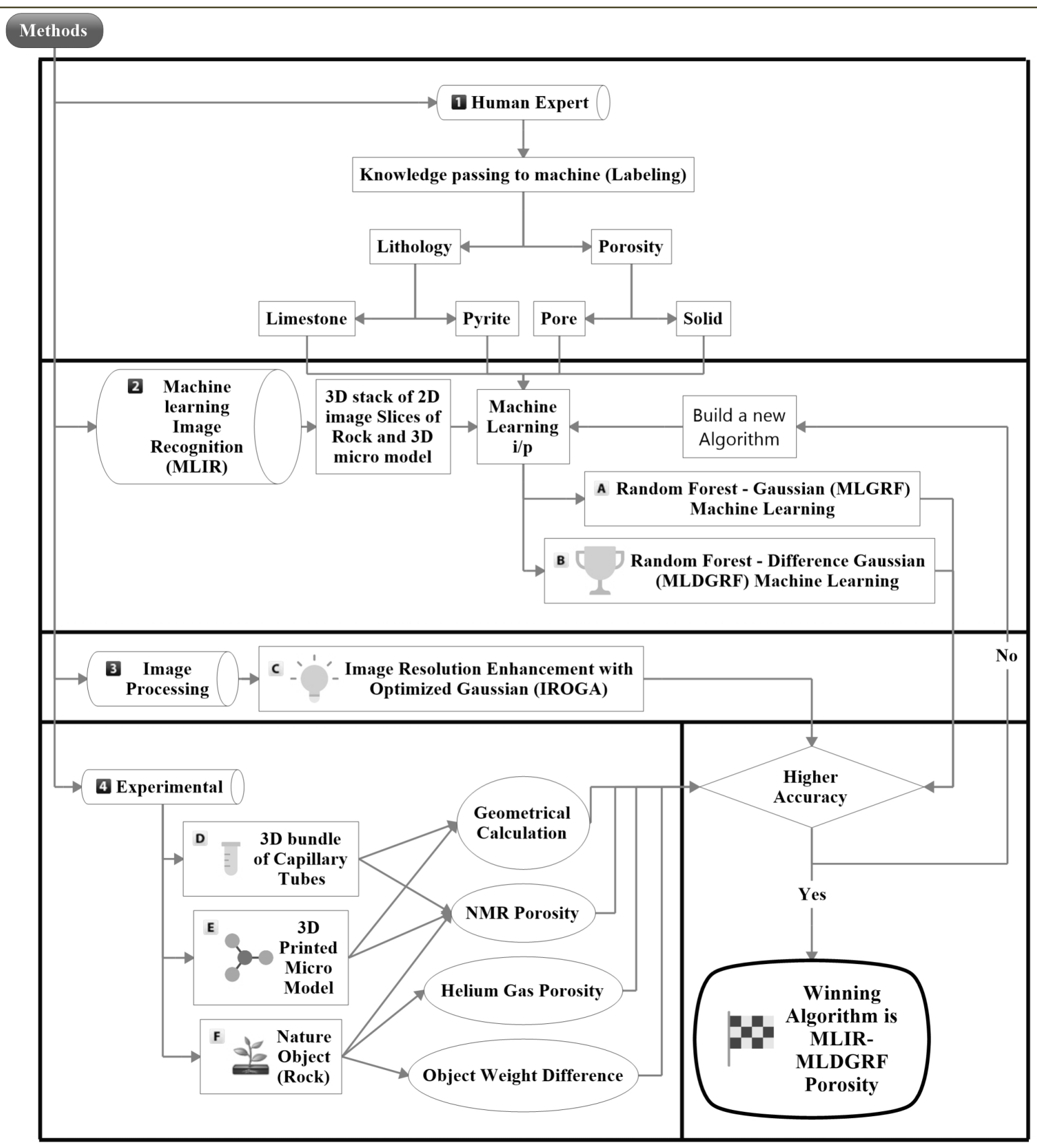

Fig. 4-Algorithms of our 3D IROGA (Image Resolution Optimized Gaussian Algorithm) and MLIR (Machine Learning Image Recognition) porosity and lithology determination and validation. 


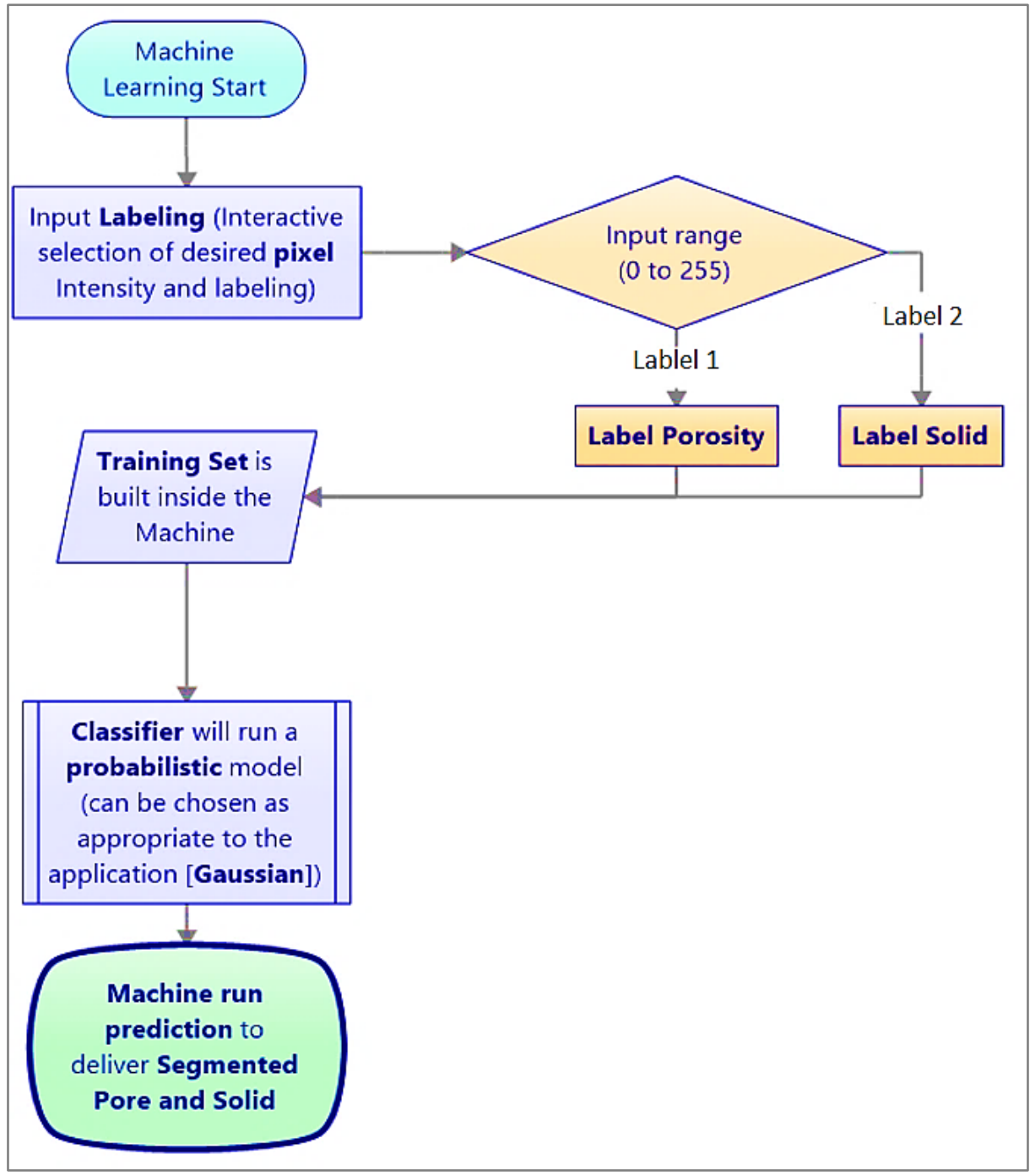

Fig. 5-Machine Learning Flow Chart.

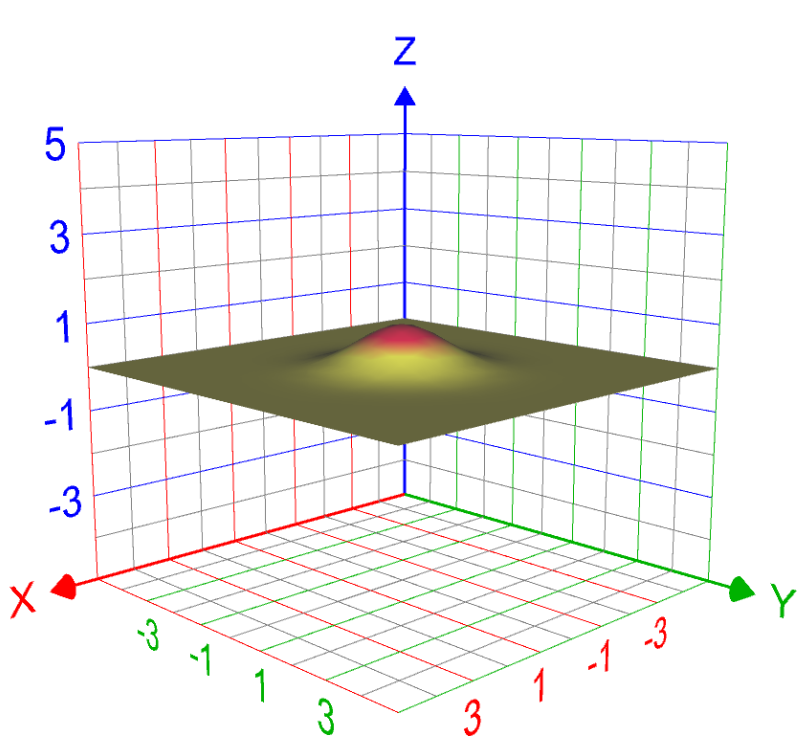

(a)

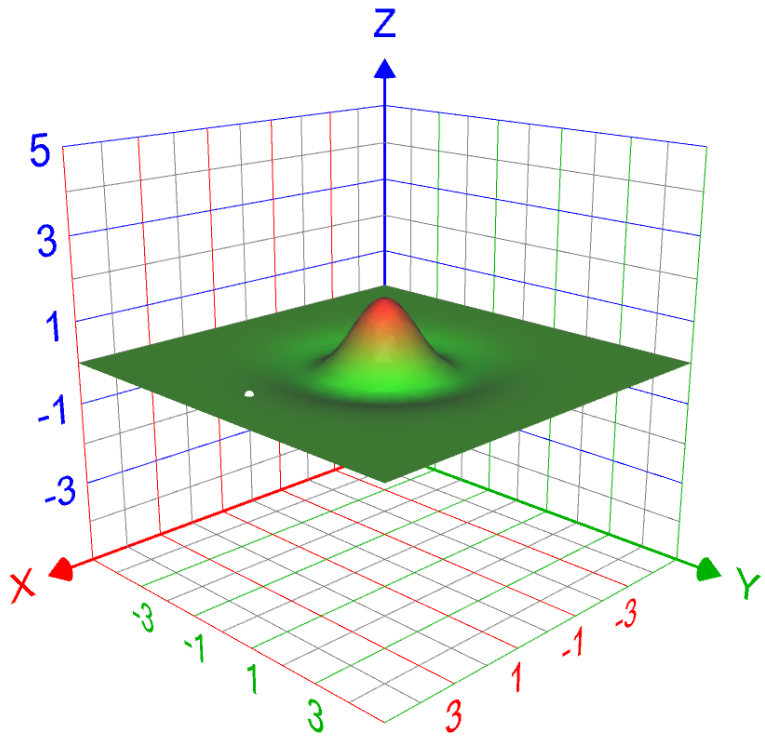

(b)

Fig. 6-A 3D Graph of (a) Gaussian Function (b) Difference of Gaussian Function. 


\section{D Micro Model Experiments and Calibration}

Without proper measurement control, the complexity of interpreting image data ( $\mu \mathrm{CT}$ and MRI) will increase. Therefore, we developed 3D Micro Model (3DMM) with various micropore sizes to train and calibrate the proposed approaches. We went through several steps to generate 3DMM, measure, and calculate, as per the following:

\section{1- 3DMM Construction}

The first thing we started with is the development of the 3DMM. In this work, we use a bundle of capillary tubes 3DMM. We built 3DMM with known dimensions of pore and solid. It consists of 26 tubes, the length is 2 inches $(50.8 \mathrm{~mm})$, while the inner diameter of each tube is $600 \mu \mathrm{m}$. The 3DMM enabled constructing and validating IROGA in a controlled environment. Fig. 7 shows 3DMM with its crosssectional and longitudinal structure. The geometrically calculated porosity of 3DMM is $7.21 \%$.

\section{2- 3DMM MRI Imaging and Porosity Calculations}

We used MRI to image 3DMM, collecting 3D MRI stack, which consists of nine 2D slices, as shown in Fig. 8. The small light dots, in Fig. 8, represent void (pore) filled with crude oil, each dot represents one tube while black represents solid objects, and the gray color rectangles represent the separators between each 2D image slice. We enlarged one of the nine MRI 2D slices, as displayed in Fig. 9.

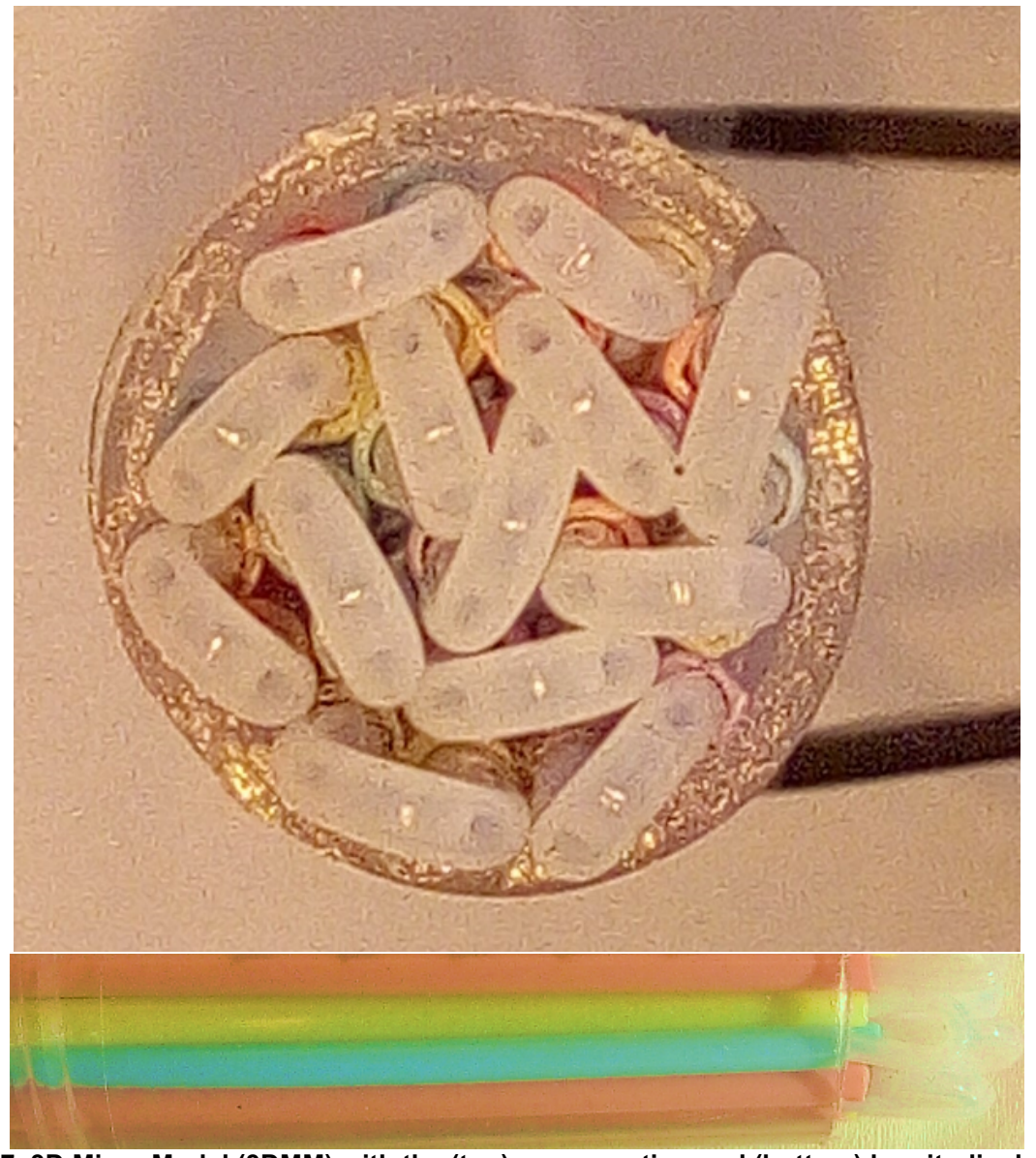

Fig. 7-3D Micro Model (3DMM) with the (top) cross section and (bottom) longitudinal section.
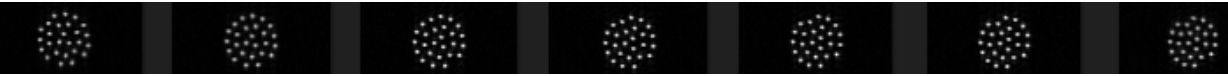

Fig. 8-3D MRI cross-section image stack of nine 2D slices for the 3D Micro Model (3DMM). 


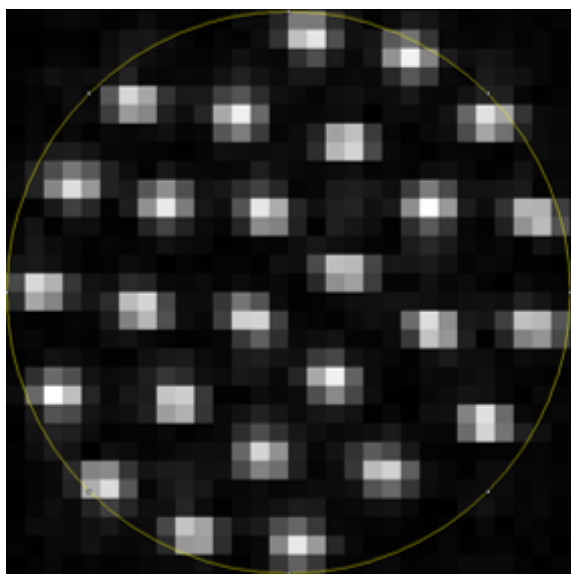

Fig. 9-Enlarged MRI 2D slice of 3D Micro Model (3DMM) shows unwanted resolution defect in gray shade.

The experiments can be used to train and calibrate our proposed methodologies as per below:

\section{3- Calibration of Image Resolution Optimized Gaussian Algorithm (IROGA)}

We designed IROGA after several verification steps. IROGA has two loops, as shown in Fig. 3, small loop, enhanced convoluted gaussian and large loop, image processing porosity determination. We constructed IROGA with continuous iterations by optimizing the gaussian values of the small loop shown in Fig. 3. While Fig. 10 is the final image of IROGA after applying the algorithm. We validated the IROGA model by calculating the porosity of 3DMM with an accuracy of $96.2 \%$. We summarized below the development of IROGA modeling:

1- We built 3DMM with $600 \mu \mathrm{m}$ inner diameter tubes (26 tubes).

2- We flooded 3DMM with crude oil collected from a lower Cretaceous Offshore Abu Dhabi formation.

3- We acquired 3D stack MRI Images with $(400 \times 400 \times 3500 \mu \mathrm{m})$ resolution for the 3DMM.

4- We structured an iterative image resolution enhancement algorithm, based on a convoluted Gaussian matrix.

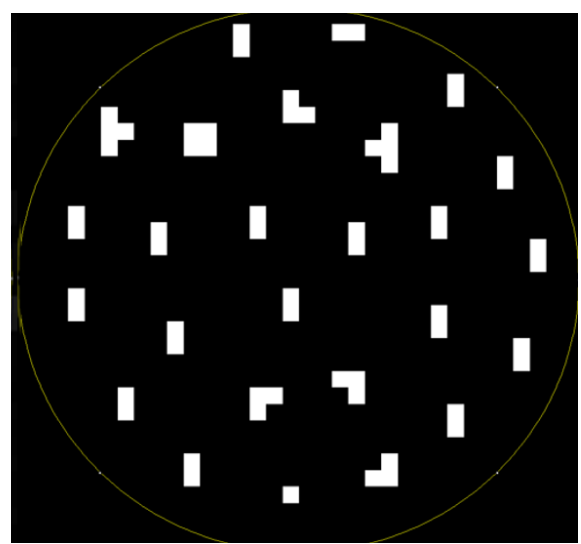

Fig.10-Cross Section of one of the 2D slices of 3DMM MRI stack after applying IROGA (Image Resolution Optimized Gaussian Algorithm).

\section{4- Calibration of Machine Learning Difference of Gaussian Random Forest (MLDGRF)}

We used the traditional Gaussian (Rasmussen 2003) Random Forest (Polan et al. 2016) as part of opensource ImageJ software (Abràmoff et al. 2004). We trained the Gaussian algorithm with data set generated via interactive labeling using human expert knowledge. Then we selected the Gaussian function to build the best representative model and perform the classification. We noticed that the void (pore area) is more significant than our reference, and the accuracy is 53.1\%, which is not acceptable. Therefore we used a different Random Forest algorithm, called Difference of Gaussian (Lindeberg 2015, Polakowski et al. 
1997, Lowe 2004) Random Forest. In Fig. 11, we see the enhanced resolution image using MLDGRF, where red color represents solid and green color represents pore. MLDGRF produced higher accuracy than IROGA to achieve $97.1 \%$, to be the best method in terms of accuracy for resolution enhancement and porosity determination. By comparing MLDGRF with IROGA porosity results, both showed proper matching to reference porosity with $97.1 \%$ and $96.2 \%$ accuracy, respectively.

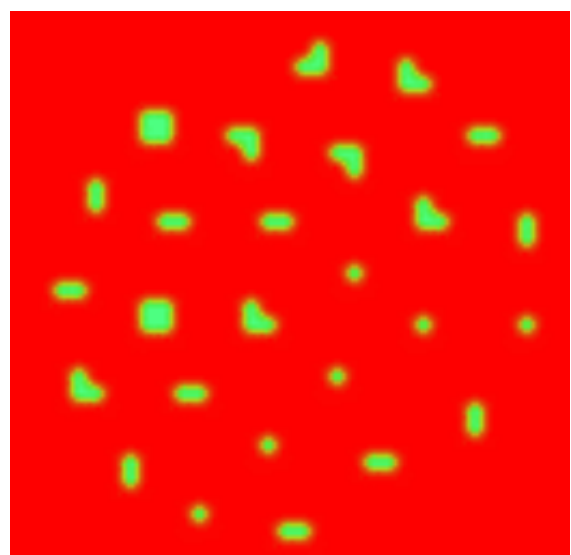

Fig. 11-3DMM MRI image after using MLDGRF (Machine Learning Difference of Gaussian Random Forest), which we found to be the best methodology.

\section{Results and Discussion}

The goal of this work, as stated earlier, is to use the physical 3DMM calibrated models for determining the porosity and lithology of natural heterogeneous carbonate rock from 3D $\mu \mathrm{CT}$ and MRI images. We measured porosity using helium gas for core plugs (Jones 1987, 1986) of the natural heterogeneous carbonate rock. Also, we imaged the same core plug with $\mu \mathrm{CT}$ and MRI (Wang, Lun, et al. 2017). We explain the steps of experimental porosity and lithology determination below:

\section{a) Natural Carbonate Rock Porosity: Experimental determination}

We measured the core plug porosity with three different measurement methods. The first measurement method is with weight difference (Luffel and Guidry 1992) of rock under dry and wet conditions. In the second method, we used NMR (Coates et al. 1999) on the same rock saturated with fluid (crude oil). The third method is made with helium gas (Jones 1987, 1986), on the same rock sample after cleaning and drying, which is the ultimate reference of porosity value.

\section{b) Natural Carbonate Rock Lithology: Experimental Determination}

Lab experiments define lithology, (Torsæter and Abtahi 2003, Lee and Chough 1987), where grain density measurement of rock core plug is essential to identify lithology. In addition to the grain density method, an X-Ray Powder Diffraction (XRPD) is another method to identify mineralogical fractions. The existence of pyrite in carbonate rock is visually recognizable to bare eyes due to its shiny metallic property. Limestone density is $\sim 2.71 \mathrm{~g} / \mathrm{cm}^{3}$, while pyrite density is $\sim 4.8 \mathrm{~g} / \mathrm{cm}^{3}$. The lab measurement showed the existence of two minerals using grain density calculation. At the same time, we also validated pyrite's existence by observing its appearance on the surface of the core plug. The higher the density of the mineral, the higher the voxel intensity of the $\mu \mathrm{CT}$ image. In our core plug, the pyrite density is much larger than that of limestone, producing identifiable shiny white regions on $\mu \mathrm{CT}$ images.

To ensure IROGA and MLDGRF methods capability to perform well with natural rock, we conducted validation tasks for both methods on lower Cretaceous heterogeneous carbonate rock. The validation results for both methods IROGA and MLIR are as per the following: 


\section{1- IROGA Porosity Validation with 3D MRI of Natural Carbonate Rock}

We performed the validation of IROGA on MRI images of carbonate core plug. Fig. 12a displays a raw MRI image of lower cretaceous carbonate rock, saturated with crude oil. Black color represents solid, and light color represents pore filled with liquid. Fig. 12b is the final MRI IROGA results in grayscale, and Fig. 12c is in color scale; the red color is solid, while the green color is pore filled with crude oil. For validating IROGA prediction, we used the reference helium gas porosity, and the resultant accuracy is $91.8 \%$.

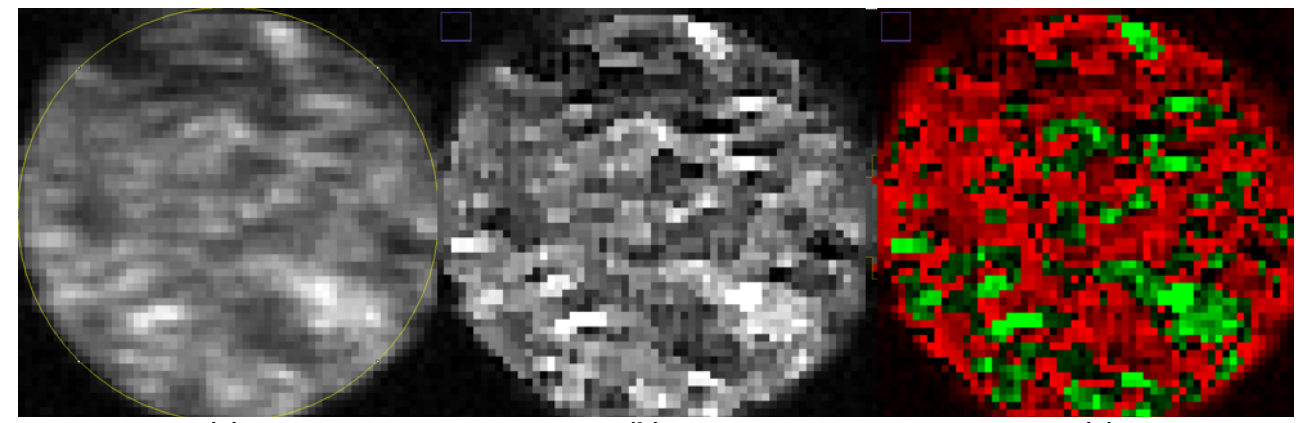

(a)

(b)

(c)

Fig. 12-Cretaceous Carbonate Rock MRI; (a) Raw image, and (b) enhanced resolution image and (c) rock and oil separation.

\section{2- MLDGRF Porosity Validation with 3D $\mu$ CT images of Natural Carbonate Rock}

Since we have demonstrated the efficiency of MLIR, especially MLDGRF, in achieving superior accuracy when measuring 3DMM porosity, we extended the proposed approach to 3D $\mu \mathrm{CT}$. We used the MLDGRF algorithm to predict 3D $\mu \mathrm{CT}$ porosity. We plotted the results of MLDGRF $\mu$ CT Porosity in Fig. 13a. Then we compared MLDGRF results with three porosity measurements, as shown in Fig. 13b. The accuracy of MLDGRF reached to $94.37 \%$. The images of 3D $\mu C T$ MLDGRF porosity prediction are in Fig. 14. The mismatch in porosity obtained from MLDGRF and the reference helium gas porosity is due to the performance of the Difference of Gaussian function parameters (Díaz-Uriarte and De Andres 2006, Boulesteix et al. 2012). Therefore, we recommend further tuning of the function parameters to increase the accuracy higher than 94.37\%. Some low-level machine learning software (i.e., Python) (Peirce 2007, Sanner 1999, Oliphant 2007) enable tuning of most of the parameters of Random Forest (Boulesteix et al. 2012), while other higher-level machine learning software facilitates only a limited number of parameters to tune. We inferred that using low-level machine learning software can help to produce higher quality results.

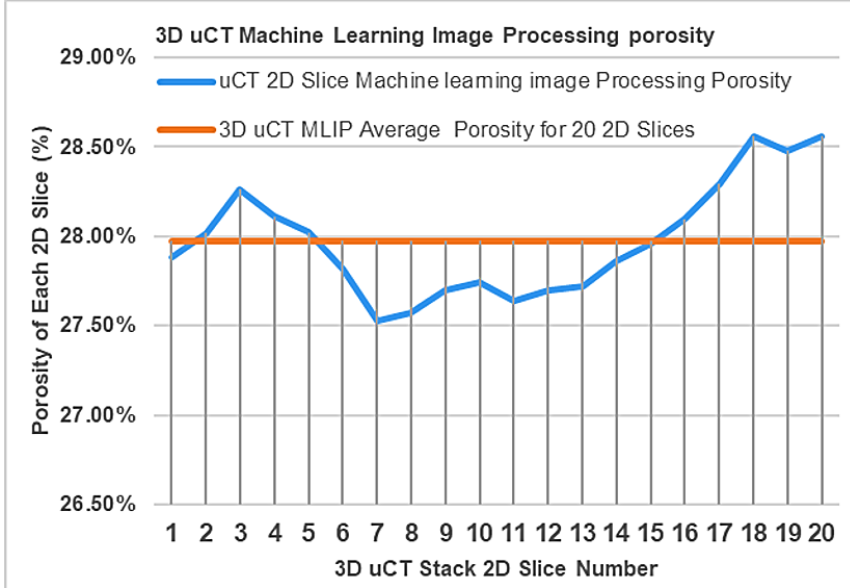

(a)

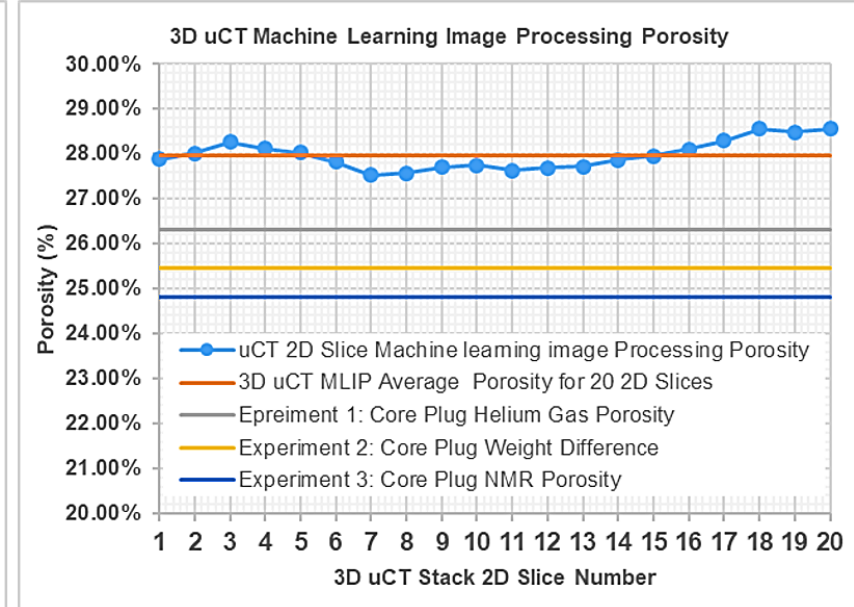

(b)

Fig. 13-Lower Cretaceous Carbonate Rock; (a) 3D $\mu$ CT MLDGRF Porosity of 20 2D slices, (b) Comparing 3D $\mu C T$ MLDGRF Porosity with reference porosities. 


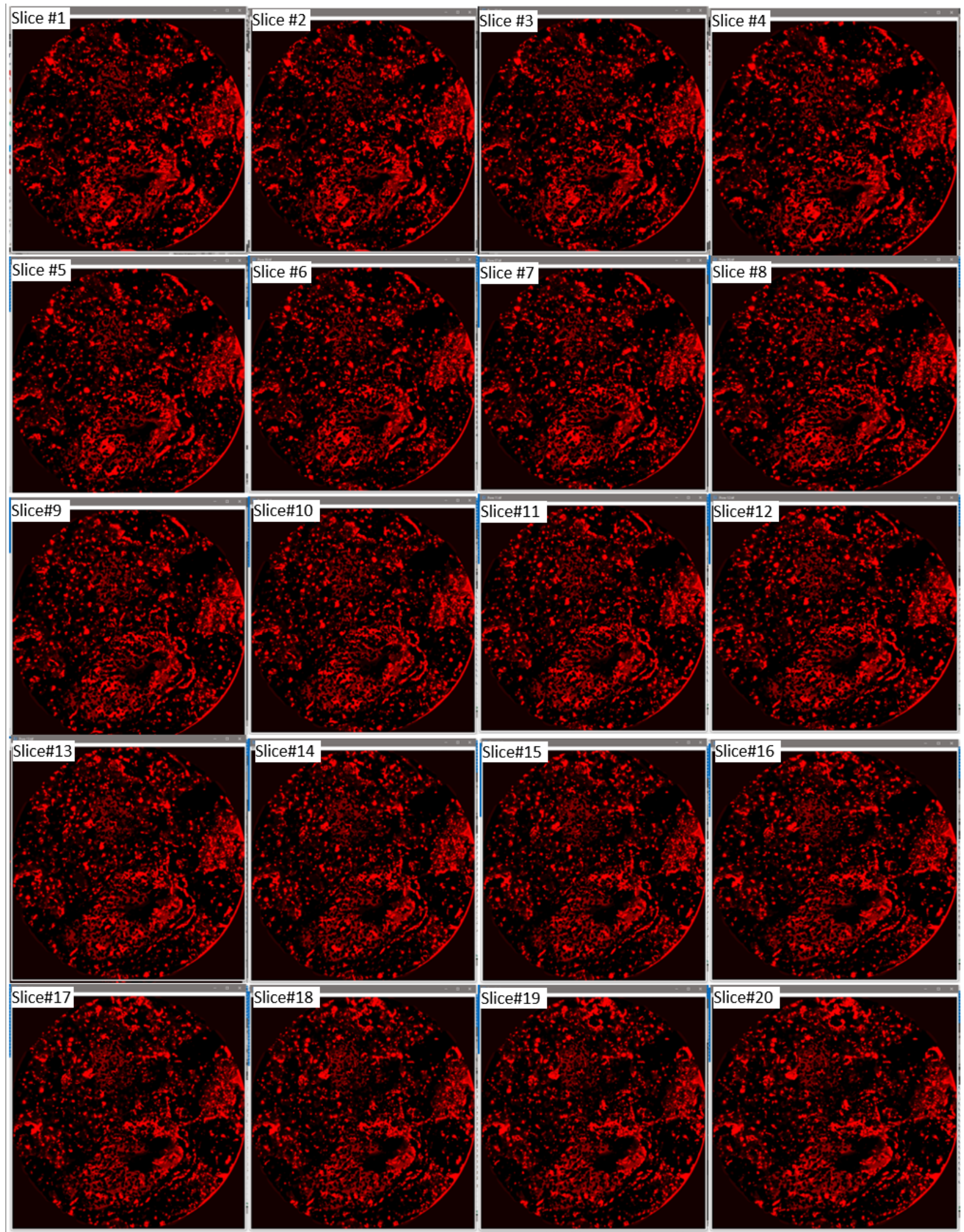

Fig. 14-The Machine Learning Image Recognition predicted Porosity (red is pore) from 3D $\mu$ CT carbonate rock image.

\section{3- MLDGRF Lithology Validation with 3D $\mu$ CT Natural Heterogeneous Carbonate Rock}

The vital step in performing rock typing as part of a reservoir characterization study is to identify lithology (Al-Farisi et al. 2002, Al-Farisi et al. 2009, Al-Farisi et al. 2013). In our research, human experts labeled different lithologies existing in $3 \mathrm{D} \mu \mathrm{CT}$ stack, in our case, Pyrite and Limestone. Then the MLDGRF algorithm is applied to differentiate the minerals in the rock, see Fig. 15. 


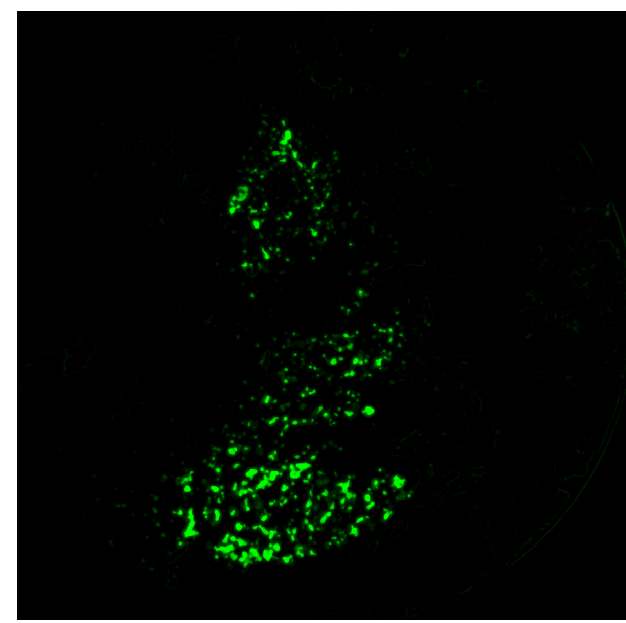

(a-Green is Pyrite)

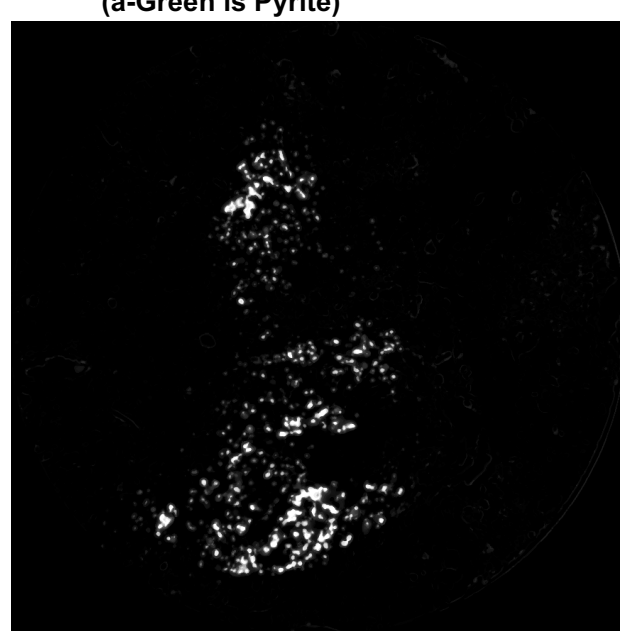

(a-White is Pyrite)

Fig. 15-Machine Learning Image Recognition Difference Gaussian Random Forest algorithm MLDGRF has identified and segmented Lithology for 3D $\mu$ CT Stack. Both (a-Green) and (a-White) are the same segmented image for Pyrite mineral, where Pyrite

is the green color in (a-Green) image as the original segmentation image, and we displayed it again in (a-White) for printing purposes if the green color is not bright. While (b-Blue) and (b-White) are similar images for Limestone segmentation, where Limestone is the blue color in (b-Blue) image as the original segmentation image, and we displayed it again in (b-White) for printing purpose if the blue color is not bright enough.

Geoscientists have performed, during the last 40 years, mineral identification from images, even for outcrop rock (Kurz et al. 2012). We noticed that most of the studies have worked on borehole imaging rather than a core plug $\mu \mathrm{CT}$ images (Knecht et al. 2003). Currently, identifying and measuring minerals in core samples are mainly performed by physical weight measurements. While in using imaging for lithology identification, either a thin section or SEM images provided the primary source for mineral analysis (He, Ding, et al. 2016). In this study, we focused on 3D $\mu$ CT images to be the source of lithology identification and quantification. Also, most of the previous work has focused on the textural identification to determine lithofacies and other petrophysical properties (i.e., Archie's equation parameters and bioclast) (Knecht et al. 2003, Ketcham and Iturrino 2005, Knackstedt et al. 2007, Knackstedt et al. 2004). The main differentiator that we used in our research for segmenting lithology was the image intensity. The image intensity of pyrite is $\sim 255$, which is white color in a grayscale image $3 \mathrm{D} \mu \mathrm{CT}$ and the highest intensity. In our rock core plug, there is no other mineral as dense as pyrite, as the other mineral is limestone.

An external commercial laboratory performed the mineralogical analysis as a reference for rock mineral composition. The mineral lab measurement technique (Torsæter and Abtahi 2003) uses helium gas for measuring void, outer volume geometry measurement, and the weight of the dry plug. This method cannot identify the location of pyrite inside the rock but can provide a value of grain density, which was 
ranging between 2.71 and $2.74 \mathrm{~g} / \mathrm{cm}^{3}$, a range that is higher than pure limestone. In another method, Xray powder diffraction (XRPD), where pyrite volume range is $0.1 \%$ to $1.1 \%$ out of total volume. While in using 3D $\mu \mathrm{CT}$, we can identify the existence and the distribution of mineral in the rock in addition to a more accurate representation of volume. The XRPD method uses powder from one part of the rock, which does not represent the whole core plug. Fig. 15 shows two different types of minerals (pyrite and limestone) that MLDGRF managed to segment and quantify. While Fig. 16 shows the distribution of pyrite inside the rock in the 3D image caption, as identified by the MLDGRF. MLDGRF found a pyrite volume of $2.4 \%$ out of total volume, as shown in Fig. 17. MLRDGF produced an accuracy of $97.7 \%$ for pyrite determination. This identification of pyrite distribution helps permeability determination from 3D $\mu \mathrm{CT}$ and further rock classification.

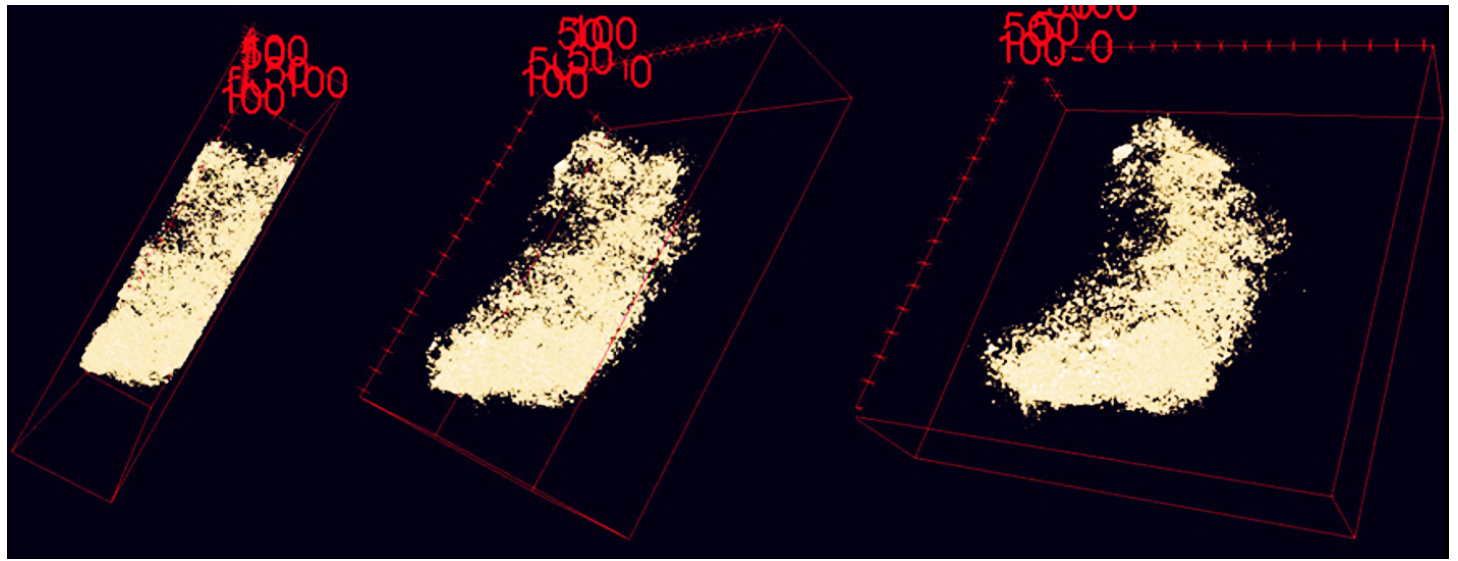

Fig. 16-A 3D view caption of segmented pyrite using Machine Learning Image Recognition Difference Gaussian Random Forest algorithm MLDGRF for 3D $\mu C T$ image Stack. The three 3D images show the same segmented pyrite from three different view angles.

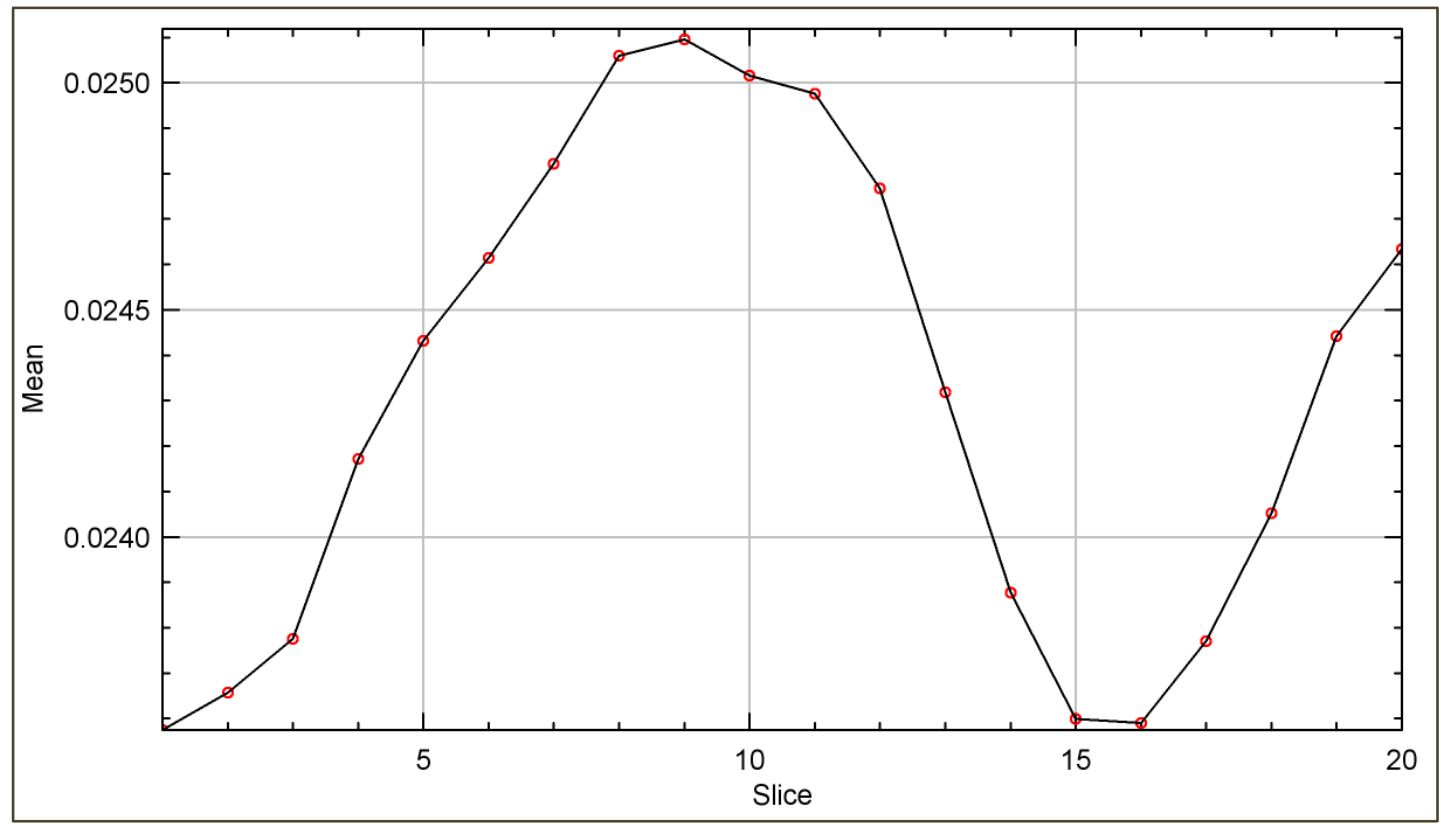

Fig. 17- Lower Cretaceous Carbonate Rock 3D $\mu$ CT MLDGRF Pyrite mean volume fraction for the 20 2D images slices.

\section{Conclusions and Recommendations}

1- To determine the reservoir properties (i.e., porosity and lithology), we investigated several methods and proposed a new one based on $\mu \mathrm{CT}$ and MRI images. We found that machine learning image recognition using the Difference of Gaussian Random Forest algorithm is superior compared to machine learning image recognition using the Gaussian Random Forest algorithm. This finding helped 
us to choose the optimal machine learning algorithm for reservoir rock properties determination from various image types. Moreover, experimental validation is necessary, as it is a physical proof and the reference to quantify the algorithm accuracy.

2- Machine Learning Difference Gaussian Random Forest algorithm works well for different types of images (i.e., $\mu \mathrm{CT}$ and MRI). If the domain expert correctly labels the desired features and chooses the best algorithm, machine learning image recognition can save years of tedious work. Therefore, machine learning is recommended in more petroleum application scenarios to produce quality results faster.

3- Improving the resolution of 3D MRI images requires adequate image processing algorithms. With experimental work, we developed a new image enhancement algorithm for binary (two classes) segmentation. Image processing can save time over machine learning for image recognition, where longer time is associated with a deeper machine learning algorithm. While for segmenting more than two classes, machine learning is better, especially with a heterogeneous rock.

4- Our work on porosity and lithology determination using machine learning has helped a better understanding of rock heterogeneity and provided insight into the analysis and digital classification of the reservoir rock.

\section{Acknowledgment}

We want to thank ADNOC, ADNOC Offshore, and Khalifa University for permitting the publication of this paper as part of a joint research project and a Ph.D. thesis. Especially we thank the support of Mr. Saoud Almehairbi, Ahmed Al-Hendi, Andreas Scheed, Ahmed Al-Riyami, Hamdan Al-Hammadi, Khalil Ibrahim and Mohamed Abdulsalam. We shall also extend our acknowledgment to Ms. Hongxia Li (Khalifa University of Science and Technology), Dr. Moustafa Dernaika (ADNOC), Mr. George Mani (Corelab), and Mr. Osama Jallad (Ingrain) for their support.

\section{References}

Abadi, Martín, Barham, Paul, Chen, Jianmin et al. 2016. Tensorflow: A system for large-scale machine learning. Proc., 12th \{USENIX\} Symposium on Operating Systems Design and Implementation ( $\{$ OSDI $\} 16) 265-283$.

Abràmoff, Michael D, Magalhães, Paulo J, and Ram, Sunanda J. 2004. Image processing with ImageJ. Biophotonics international 11 (7): 36-42.

Al-Farisi, Omar, Belhaj, Hadi, Ghedan, Shawket et al. 2013. Carbonate Rock Types Matrix, the Ultimate Rock Properties Classification Catalogue. Proc., International Petroleum Technology Conference.

Al-Farisi, Omar, Elhami, Mohamed, Al-Felasi, Ali et al. 2009. Revelation of carbonate rock typing-the resolved gap. Proc., SPE/EAGE Reservoir Characterization \& Simulation Conference.

Al-Farisi, OU, Belgaied, AA, Shebl, HT et al. 2002. Well Logs: The Link Between Geology and Reservoir Performance. Abstract Geo2002 96.

Alqahtani, Naif, Armstrong, Ryan T, and Mostaghimi, Peyman. 2018. Deep learning convolutional neural networks to predict porous media properties. Proc., SPE Asia Pacific oil and gas conference and exhibition.

Arganda-Carreras, Ignacio, Kaynig, Verena, Rueden, Curtis et al. 2017. Trainable Weka Segmentation: a machine learning tool for microscopy pixel classification. Bioinformatics 33 (15): 2424-2426.

Ausbrooks, Robin, Hurley, Neil F, May, Andrew et al. 1999. Pore-size distributions in vuggy carbonates from core images, NMR, and capillary pressure. Proc., SPE annual technical conference and exhibition.

Boulesteix, Anne-Laure, Janitza, Silke, Kruppa, Jochen et al. 2012. Overview of random forest methodology and practical guidance with emphasis on computational biology and bioinformatics. Wiley Interdisciplinary Reviews: Data Mining and Knowledge Discovery 2 (6): 493-507.

Bovik, Alan C. 2009. The essential guide to image processing: Academic Press. 
Breiman, Leo. 2001. Random forests. Machine learning 45 (1): 5-32.

Buades, Antoni, Coll, Bartomeu, and Morel, J-M. 2005. A non-local algorithm for image denoising. Proc., 2005 IEEE Computer Society Conference on Computer Vision and Pattern Recognition (CVPR'05)60-65.

Calliess, Jan-P, Mai, Michael, and Pfeiffer, Sebastian. 2012. On the computational benefit of tensor separation for high-dimensional discrete convolutions. Multidimensional Systems and Signal Processing 23 (1-2): 255-279.

Chen, E-Liang, Chung, Pau-Choo, Chen, Ching-Liang et al. 1998. An automatic diagnostic system for CT liver image classification. IEEE transactions on biomedical engineering 45 (6): 783-794.

Chhatre, Shreerang S, Sahoo, Hemant, Leonardi, Sergio et al. 2017. A Blind Study of Four Digital Rock Physics Vendor Labs on Porosity, Absolute Permeability, and Primary Drainage Capillary Pressure Data on Tight Outcrop Rocks. Proc., Oral presentation given at the Annual Symposium of the Society of Core Analysts, Vienna, Austria.

Coates, George R, Xiao, Lizhi, and Prammer, Manfred G. 1999. NMR logging: principles and applications, Vol. 234: Haliburton Energy Services Houston.

Collewet, G, Strzelecki, M, and Mariette, F. 2004. Influence of MRI acquisition protocols and image intensity normalization methods on texture classification. Magnetic resonance imaging 22 (1): 8191.

Collins, Tony J. 2007. ImageJ for microscopy. Biotechniques 43 (S1): S25-S30.

Decencière, Etienne, Cazuguel, Guy, Zhang, Xiwei et al. 2013. TeleOphta: Machine learning and image processing methods for teleophthalmology. Irbm 34 (2): 196-203.

Deriche, Rachid. 1987. Using Canny's criteria to derive a recursively implemented optimal edge detector. International journal of computer vision 1 (2): 167-187.

Dernaika, Moustafa, Al Mansoori, Maisoon, Singh, Maniesh et al. 2018. Digital and Conventional Techniques to Study Permeability Heterogeneity in Complex Carbonate Rocks. Petrophysics 59 (03): 373-396.

Díaz-Uriarte, Ramón and De Andres, Sara Alvarez. 2006. Gene selection and classification of microarray data using random forest. BMC bioinformatics 7 (1): 3.

Dietterich, Thomas G. 2000. Ensemble methods in machine learning. Proc., International workshop on multiple classifier systems 1-15.

Dong, $\mathrm{Hu}$ and Blunt, Martin J. 2009. Pore-network extraction from micro-computerized-tomography images. Physical review E 80 (3): 036307.

Drexler, Wolfgang, Morgner, Uwe, Ghanta, Ravi K et al. 2001. Ultrahigh-resolution ophthalmic optical coherence tomography. Nature medicine 7 (4): 502.

Dunlavy, Daniel, Kolda, Tamara G, and Kegelmeyer, W Philip. 2008. Tensor Decompositions for Analyzing Multi-link Graphs, Sandia National Lab.(SNL-NM), Albuquerque, NM (United States); Sandia ....

Ehrlich, Robert, Kennedy, Stephen K, Crabtree, Sterling J et al. 1984. Petrographic image analysis; I, Analysis of reservoir pore complexes. Journal of Sedimentary Research 54 (4): 1365-1378.

Ferreira, Tiago and Rasband, Wayne. 2012. ImageJ user guide. ImageJ/Fiji 1: 155-161.

Gerke, Kirill M, Karsanina, Marina V, and Mallants, Dirk. 2015. Universal stochastic multiscale image fusion: an example application for shale rock. Scientific reports 5: 15880.

Gonzalez, Rafael C, Woods, Richards E, and Eddins, Steven L. 2009. Digital processing using MATLAB, Gatesmark Publishing, Knoxville (Reprint).

Goodfellow, I. 2016. Deep Learning/Ian Goodfellow, Yoshua Bengio, Aaron Courville, MIT Press (Reprint).

He, Jianhua, Ding, Wenlong, Li, Ang et al. 2016. Quantitative microporosity evaluation using mercury injection and digital image analysis in tight carbonate rocks: A case study from the Ordovician in the Tazhong Palaeouplift, Tarim Basin, NW China. Journal of Natural Gas Science and Engineering 34: 627-644. 
He, Kaiming, Zhang, Xiangyu, Ren, Shaoqing et al. 2016. Deep residual learning for image recognition. Proc., Proceedings of the IEEE conference on computer vision and pattern recognition770-778.

Huang, Bo, Wang, Wenqin, Bates, Mark et al. 2008. Three-dimensional super-resolution imaging by stochastic optical reconstruction microscopy. Science 319 (5864): 810-813.

Jones, Stanley C. 1986. System for measuring the pore volume and permeability of very tight core plugs and method therefor, Google Patents (Reprint).

Jones, Stanley C. 1987. Porous end plug disk for testing core samples, Google Patents (Reprint).

Kann, Benjamin H, Thompson, Reid, Thomas Jr, Charles R et al. 2019. Artificial Intelligence in Oncology: Current Applications and Future Directions. Oncology 33 (2).

Ketcham, Richard A and Iturrino, Gerardo J. 2005. Nondestructive high-resolution visualization and measurement of anisotropic effective porosity in complex lithologies using high-resolution X-ray computed tomography. Journal of Hydrology 302 (1-4): 92-106.

Knackstedt, MA, Arns, CH, Limaye, Ajay et al. 2004. Digital Core Laboratory: Properties of reservoir core derived from 3D images. Proc., SPE Asia Pacific Conference on Integrated Modelling for Asset Management.

Knackstedt, MA, Arns, CH, Sheppard, AP et al. 2007. Archie's exponents in complex lithologies derived from 3D digital core analysis. Proc., 48th Annual Logging Symposium.

Knecht, Leonora, Mathis, Benoit, Leduc, Jean-Pierre et al. 2003. Electrofacies and permeability modeling in carbonate reservoirs using image texture analysis and clustering tools. Proc., SPWLA 44th annual logging symposium.

Kotsiantis, Sotiris B, Zaharakis, I, and Pintelas, P. 2007. Supervised machine learning: A review of classification techniques. Emerging artificial intelligence applications in computer engineering 160: 3-24.

Kurz, Tobias H, Dewit, Julie, Buckley, Simon J et al. 2012. Hyperspectral image analysis of different carbonate lithologies (limestone, karst and hydrothermal dolomites): the Pozalagua Quarry case study (Cantabria, North-west Spain). Sedimentology 59 (2): 623-645.

Lee, HJ and Chough, SK. 1987. Bulk density, void ratio, and porosity determined from average grain density and water content: an evaluation of errors.

Li, Can, Hu, Miao, Li, Yunning et al. 2018. Analogue signal and image processing with large memristor crossbars. Nature Electronics 1 (1): 52.

Li, Huiqi and Chutatape, Opas. 2004. Automated feature extraction in color retinal images by a model based approach. IEEE Transactions on biomedical engineering 51 (2): 246-254.

Lindeberg, Tony. 2015. Image matching using generalized scale-space interest points. Journal of Mathematical Imaging and Vision 52 (1): 3-36.

Lindquist, W Brent, Lee, Sang-Moon, Coker, David A et al. 1996. Medial axis analysis of void structure in three-dimensional tomographic images of porous media. Journal of Geophysical Research: Solid Earth 101 (B4): 8297-8310.

Lindquist, WB and Venkatarangan, A. 1999. Investigating 3D geometry of porous media from high resolution images. Physics and Chemistry of the Earth, Part A: Solid Earth and Geodesy 24 (7): 593-599.

Liu, Zhe-Yu, Li, Yi-Qiang, Cui, Ming-Hui et al. 2016. Pore-scale investigation of residual oil displacement in surfactant-polymer flooding using nuclear magnetic resonance experiments. Petroleum Science 13 (1): 91-99.

Lowe, David G. 2004. Distinctive image features from scale-invariant keypoints. International journal of computer vision 60 (2): 91-110.

Luffel, DL and Guidry, FK. 1992. New core analysis methods for measuring reservoir rock properties of Devonian shale. Journal of Petroleum Technology 44 (11): 1,184-1,190.

Malcolm, AA, Leong, HY, Spowage, AC et al. 2007. Image segmentation and analysis for porosity measurement. Journal of materials Processing technology 192: 391-396.

Marr, Bernard. Will These 4 Skills Help Future-Proof Your Career?, 
https://www.forbes.com/sites/bernardmarr/2016/08/12/will-these-4-skills-help-future-proofyour-career/\#46ed523cc0b1.

Marschall, D, Gardner, JS, Mardon, D et al. 1995. Method for correlating NMR relaxometry and mercury injection data. Proc., 1995 SCA Conference, paper9511.

Mowers, Theodore T and Budd, David A. 1996. Quantification of porosity and permeability reduction due to calcite cementation using computer-assisted petrographic image analysis techniques. $A A P G$ bulletin 80 (3): 309-321.

Müller, Andreas C and Guido, Sarah. 2016. Introduction to machine learning with Python: a guide for data scientists: " O'Reilly Media, Inc.".

Oliphant, Travis E. 2007. Python for scientific computing. Computing in Science \& Engineering 9 (3): 10-20.

Pedregosa, Fabian, Varoquaux, Gaël, Gramfort, Alexandre et al. 2011. Scikit-learn: Machine learning in Python. Journal of machine learning research 12 (Oct): 2825-2830.

Peirce, Jonathan W. 2007. PsychoPy—psychophysics software in Python. Journal of neuroscience methods 162 (1-2): 8-13.

Phillips, P Jonathon and O'toole, Alice J. 2014. Comparison of human and computer performance across face recognition experiments. Image and Vision Computing 32 (1): 74-85.

Polakowski, William E, Cournoyer, Donald A, Rogers, Steven K et al. 1997. Computer-aided breast cancer detection and diagnosis of masses using difference of Gaussians and derivative-based feature saliency. IEEE transactions on medical imaging 16 (6): 811-819.

Polan, Daniel F, Brady, Samuel L, and Kaufman, Robert A. 2016. Tissue segmentation of computed tomography images using a Random Forest algorithm: a feasibility study. Physics in Medicine \& Biology 61 (17): 6553.

Rafael, Gonzalez C, Richard, Woods E, and Steven, Eddins L. 2007. Digital image processing 3rd edition. Eighth Impression.

Rasmussen, Carl Edward. 2003. Gaussian processes in machine learning. Proc., Summer School on Machine Learning63-71.

Russ, John C. 2016. The image processing handbook: CRC press.

Sánchez, Clara I, Hornero, Roberto, López, María I et al. 2008. A novel automatic image processing algorithm for detection of hard exudates based on retinal image analysis. Medical engineering \& physics 30 (3): 350-357.

Sanner, Michel F. 1999. Python: a programming language for software integration and development. $J$ Mol Graph Model 17 (1): 57-61.

Saxena, Nishank, Mavko, Gary, Hofmann, Ronny et al. 2017. Estimating permeability from thin sections without reconstruction: Digital rock study of 3D properties from 2D images. Computers \& Geosciences 102: 79-99.

Schindelin, Johannes, Arganda-Carreras, Ignacio, Frise, Erwin et al. 2012. Fiji: an open-source platform for biological-image analysis. Nature methods 9 (7): 676.

Shattuck, David W, Sandor-Leahy, Stephanie R, Schaper, Kirt A et al. 2001. Magnetic resonance image tissue classification using a partial volume model. NeuroImage 13 (5): 856-876.

Simonyan, Karen and Zisserman, Andrew. 2014. Very deep convolutional networks for large-scale image recognition. arXiv preprint arXiv: 14091556.

Singh, Kamaljit, Menke, Hannah, Andrew, Matthew et al. 2017. Dynamics of snap-off and pore-filling events during two-phase fluid flow in permeable media. Scientific reports 7 (1): 5192.

Sommer, Christoph, Straehle, Christoph, Koethe, Ullrich et al. 2011. Ilastik: Interactive learning and segmentation toolkit. Proc., 2011 IEEE international symposium on biomedical imaging: From nano to macro230-233.

Torsæter, Ole and Abtahi, Manoochehr. 2003. Experimental reservoir engineering laboratory workbook. Department of Petroleum Engineering and Applied Geophysics, Norwegian University of Science and Technology (NTNU), Trondheim. 
Wang, Haitao, Lun, Zengmin, Lv, Chengyuan et al. 2017. Measurement and visualization of tight rock exposed to CO 2 using NMR relaxometry and MRI. Scientific reports 7: 44354.

Wang, Yunzhi, Aghaei, Faranak, Zarafshani, Ali et al. 2017. Computer-aided classification of mammographic masses using visually sensitive image features. Journal of X-ray Science and technology 25 (1): 171-186.

Whitney, Lance. 2017. Are computers already smarter than humans? Time Magazine, available at: http://time com/4960778/computers-smarter-than-humans/(accessed 23 March 2018)[Google Scholar].

Wilson, Kevin. 2014. Microsoft office 365. In Using office 365, 1-14. Springer.

$\mathrm{Xu}$, Hao, Tang, Dazhen, Zhao, Junlong et al. 2015. A precise measurement method for shale porosity with low-field nuclear magnetic resonance: A case study of the Carboniferous-Permian strata in the Linxing area, eastern Ordos Basin, China. Fuel 143: 47-54.

Yang, Jianchao, Wright, John, Huang, Thomas S et al. 2010. Image super-resolution via sparse representation. IEEE transactions on image processing 19 (11): 2861-2873.

Yu, Ruiyun, Yang, Yu, Yang, Leyou et al. 2016. RAQ-a random forest approach for predicting air quality in urban sensing systems. Sensors 16 (1): 86.

Zhang, Yan, Passmore, Peter J, and Bayford, Richard H. 2006. Visualization and Post-processing of 5D brain images. Proc., 2005 IEEE Engineering in Medicine and Biology 27th Annual Conference1083-1086.

Zhou, Qi You, Shimada, Jun, and Sato, Akira. 2001. Three-dimensional spatial and temporal monitoring of soil water content using electrical resistivity tomography. Water Resources Research 37 (2): 273-285. 